EFFECT OF VARIOUS INHALED VAPORS ON RESPIRATION

AND BLOOD PRESSURE IN ANESTHETIZED, UNANESTHETIZED, SLEEPING AND ANOSMIC SUBJECTS

\author{
WILLIAM F. ALLEN
}

From the Department of Anatomy of the University of Oregon Medical School, Portland

Reprinted from The American Journal of Physiology

Vol. 88, No. 4, May, 1929 
Reprinted from The American Journal of Physiodogr

Vol. 88, No. 4, May, 1929

\title{
EFFECT OF VARIOUS INHALED VAPORS ON RESPIRATION AND BLOOD PRESSURE IN ANESTHETIZED, UNANES- THETIZED, SLEEPING AND ANOSMIC SUBJECTS
}

\author{
WILLIAM F. ALLEN \\ From the Department of Anatomy of the University of Oregon Medical School, Portland
}

Received for publication February 11, 1929

The subjects of these experiments were normal individuals, an anosmic and selected hospital patients under light ether anesthesia. The normal subjects and the anosmic were students who had done some laboratory experimentation and were willing to coopperate in every way. With this type of subjects the psychic impulses of fear and surprise were very well eliminated and all other psychic phenomena were excluded in so far as possible. With the exception of the anosmic all of the subjects were fully up to the average in the functional activity of the olfactory and trigeminal systems. All of the tests on the non-anesthetized subjects were conducted during the summer months when they were comparatively free from infections of the upper respiratory tract.

Technical procedure. The various vapors were inhaled from cones held below the nostrils, the distance varying with the nature of the substance. All subjects, not under an anesthetic or asleep, were blindfolded and their ears were stopped with cotton. Continuous tracings were recorded during the time a subject was inhaling from a blank cone and from a second cone containing the odoriferous or irritating substance. An assistant manipulated the signal magnet by observing the time when the second cone was substituted for the blank cone and the time when the cone containing the test substance was withdrawn. If an inhalant proved too irritating the subject signaled by a movement of the thumb. After a test had been recorded the subject was asked to describe the sensation and if possible to name the vapor inhaled.

Thoracic-respiratory tracings were obtained after the manner described in the first report of a previous series. A glass tambour strapped over the carotid artery and connected with a Marey's tambour by tubing served to record the carotid pulse. The systolic blood pressure was taken with a sphygmomanometer from the forearm of the subject for a period of a minute. During the first thirty seconds several systolic readings were taken at the time a blank cone was being held below the nostrils of the subject who was blindfolded, and a like number of readings were taken during the 
remaining thirty seconds in which the subject was inhaling the test substance.

This method obviously involves the personal equation of the individual recording the blood pressure. In every subject some of the readings were checked by two individuals. Doctor Baird and Mr. Straumfjord took simultaneous readings from the right and left arms of the anosmatic subject. Neither recorder knew the inhalant used and each wrote his readings independently of the other. Upon comparing these data and other data it would appear that any change of the systolic pressure, amounting to $2 \mathrm{~mm}$. or more during the inhalation of a vapor is of significance, but anything less than $2 \mathrm{~mm}$, is entirely within the limits of normal records independent of inhalants.

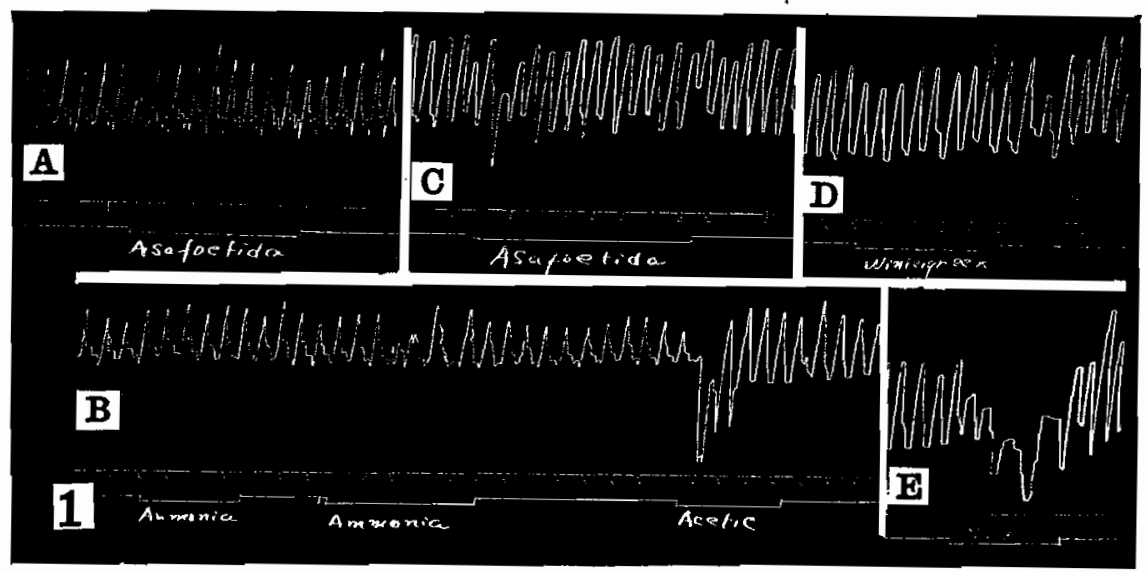

Fig. 1. Thoracic respiratory tracings, inspiration upstroke, time in 5 seconds; tracings $A$ and $B$ from normal subject $A$ asleep, tracings $C$ to $E$ from normal subject A awake.

The writer desires at this time to express his thanks to Doctor Manville, Doctor Baird, Mr. Straumfjord and Mr. Schwichtenberg for taking these readings.

Respiratory tests on normal subject A. asleep and awake. The first part of this experiment is concerned with the effect or various vapors on the thoracic respiration of my 18 year old son when he was sound asleep. Numerous tracings taken while he was asleep demonstrate that respiration had taken place at the regular rate of a little less than 18 excursions a minute. There is, however, some irregularity in the amplitude of these excursions, in that occasionally one would be short. A comparison of the tracings obtained with oil of cloves, orange, rose, lavender, bergamot, butyric acid, asafetida (fig. $1 \mathrm{~A}$ ), menthol, eucalyptus, camphor, xylol, 
benzol, ether and chloroform with the preceding normal excursions reveals no more alteration of respiration than that which occurs during normal respiration. The same negative results were obtained on repeating the foregoing tests. As will be shown in the second part of these tests, when the subject was awake, the inhalation of asafetida produced a pronounced effect on respiration, so that this substance was inhaled frequently during the sleeping tests to determine whether the lad was actually asleep.

To the left in figure $1 \mathrm{~B}$ ammonia vapor of strong concentration was inhaled for 22 seconds without awakening the subject or altering his respiration. Fifteen seconds later ammonia was inhaled for the second time for a period of 25 seconds. This time $(B$, center) there is a marked depression of two inspirations, a deepening of the next two, a rapid return to normal upon removal of the inhalant and the boy was partially awakened. As a result of inhaling acetic acid (fig. $1 \mathrm{~B}$, right) the first inspiration is stopped immediately and followed by several deep expirations. After this stimulation the return to normal includes several deep inspirations. This vapor woke up the subject completely.

During the second part of this experiment the above mentioned vapors were inhaled by subject A. fully awake. These tracings and others made two days later with the boy awake show that inhalation of the agreeable odors-oil of cloves and bergamot-produces a lowered inspiratory phase of the first few excursions without any change in their rate. Tracings from the disagreeable odors asafetida (fig. $1 \mathrm{C}$ ), butyric acid and fresh cat's urine reveal very irregular excursions during the interval of stimulation, some showing a deepened exhalation phase and others a considerably depressed inspiratory phase, their rate remaining unchanged. The xylol and alcohol graphs disclose a lengthened inspiratory stage and no change in the rate of excursion. Tracings obtained during the inhalation of wintergreen (fig. 1 D), camphor, menthol, ether, chloroform and pyridin demonstrate a tendency throughout stimulation toward both a shortening and lengthening of the inspiratory phase of the excursions, chiefly a depression. Some of these substances, ether, chloroform and benzol also produce considerable deepening of the expiratory phase of the excursions. Respiratory excursions in the records obtained at the time of inhalation of the very irritating vapors from oil of mustard, acetic acid and ammonia (fig. $1 \mathrm{E}$ ) always portray a marked shortening of the inspiratory phase, some increase in the expiratory phase and a reduction in rate of excursion or a complete arrest of respiration.

Respiratory tests in normal subjects $C$., $S$. and $W$. Of these three individuals, W. is the most sensitive and reacts strongest to the mild agreeable odors which are specific olfactory stimuli for rabbits. The respiratory excursions in the tracings obtained from C. and W. at the time of inhalating bergamot, oil of cloves (fig. $2 \mathrm{~A}$ ) and lavender exhibit a considerably depressed 
inspiratory phase and some increase in number; while the excursions from $\mathrm{S}$. portray a lengthened inspiratory phase and a decrease in number, the actual rate of movement appears to be unchanged. The effect of the disagreeable odors-asafetida (fig. $2 \mathrm{~B}$ from $\mathrm{W}$ ) and butyric acid in all three subjects is one which shortens the inspiratory phase and increases the number of excursions. Wintergreen produces a marked depression in C. and $W$., while in one set of tracings in $\mathrm{S}$. it evokes a deepening of the inspirations and in a second set of tracings there is a marked depression of the first four or five inspirations and a deepening of the remainder. The excursions in the tracings for the mild irritants and odors-camphor, menthol, eucalyptus, peppermint, ether, chloroform, alcohol, xylol, benzol (fig. $2 \mathrm{C}$.

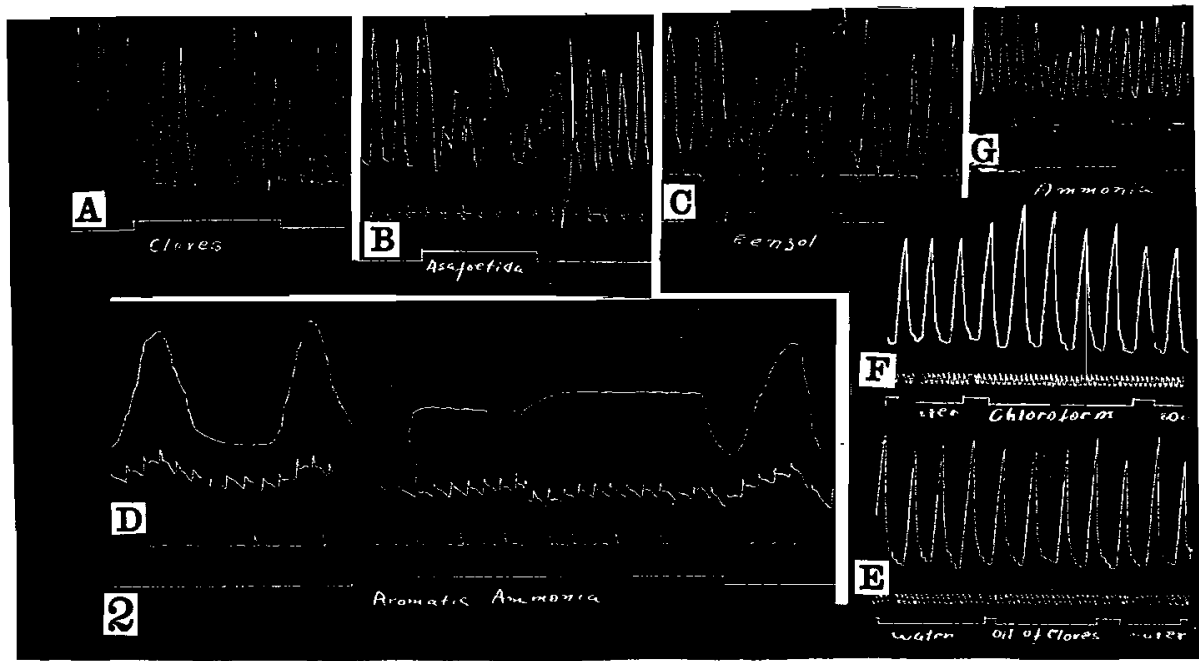

Fig. 2. Same as figure 1; tracings $A$ to $D$ from normal subject $W$, the tracing below the respiratory tracing in $\mathrm{D}$ is from the carotid artery; tracings $\mathrm{E}$ to $\mathrm{G}$ are from the anosmic.

from W.) and phenol reveal a considerably lowered inspiratory stage, frequently an increased expiratory stage and usually an increased number of excursions in $\mathrm{C}$. and W.; while in $\mathrm{S}$. the excursions are lengthened in both the inspiratory and expiratory stages. As a result of inhalation of strong concentrations of the very irritating vapors-oil of mustard, acetic acid and ammonia (fig. $2 \mathrm{D}$ from $\mathrm{W}$, upper tracing) there is a marked depression and slowing of the excursions or complete suppression of respiration in all three subjects.

In those records where considerable suppression of ventilation has taken place during the stimulation period, removal of the inhalant is followed by a considerable increase in ventilation. This may be produced by an acceleration or a deepening of the respiratory movements or both (fig. $1 \mathrm{E}$ ). 
Some respiratory graphs were obtained from a small boy who was said to have a very acute sense of smell. The lad proved to be such an excitable chap that the large number of body movements recorded made these tracings worthless.

Respiratory tests in the anosmic subject $H$. This student has had no sense of smell for seven years as the result of a skull fracture caused by a falling telephone pole. A description of the fracture from x-ray plates by Drs. A. E., Paul and Eugene Rockey to the Oregon Accident Commission is as follows: "A comminuted fracture appears on the left side of the skull, consisting of two horizontal fractures in the temporal region which join a vertical fracture. The fracture lines extend into the vault of the pharynx and through the sphenoid and ethmoid sinuses." Bleeding was described from the nose and the left ear and there was a paralysis of the left rectus lateralis eye muscle. As a result of this accident the patient completely lost the sense of smell. The following tests, however, reveal that at least a branch of the trigeminal nerve to the nasal mucosa is functioning.

Tabulated data from two sets of tracings taken on different days demonstrate that inhalation of the agreeable and disagreeable odors-oil of cloves (fig. $2 \mathrm{E}$ ), orange, rose, lavender, bergamot, asafetida, butyric acid and fresh cat's urine produce no change in respiration. Xylol and wintergreen also gave negative results. The following mild irritants and odorsmenthol, eucalyptus, camphor, peppermint, ether, chloroform (fig. $2 \mathrm{~F}$ ) and benzol produce a considerable augmentation of the inspirations, and the amount of reduction in the rate of the excursions suggests that the actual rate of respiratory movement is unchanged. Weak concentrations of the strongly irritating substances, such as formalin, acetic acid and ammonia yield similar reactions to the mild irritants; while strong concentrations of the same substances (fig. $2 \mathrm{G}$, ammonia graph) cause the excursions to show a marked depression of their inspiratory phase and some increase in rate. If strong concentrations of mustard are to elicit any change in the respiration of this subject the cone must be held close to the nostrils. The effect which comes on slowly is one which increases the depth of both inspirations and expirations, together with some decrease in the rate of excursion.

After a respiratory tracing had been obtained the anosmic was asked to describe the effect of the substance. His answers show that none of the agreeable or disagreeable odors were perceived. Fresh cat's urine was not detected, but old decomposed cat's urine, possessing a trace of ammonia, was said to produce a different sensation than a strong concentration of ammonia did. Oil of wintergreen, camphor, menthol, eucalyptus, xylol and benzol were not detected, though some of these substances altered his respiration. Pyridin was described as a taste sensation coming from the back of the throat and tongue. Peppermint caused a cooling sensation in 
the nose and ether a disagreeable burning sensation appearing quickly. On the other hand, chloroform elicited a pleasant sensation in the nose. Formalin, described as a tickling sensation in the nose, was a long time in appearing. A weak concentration of acetic acid or ammonia produced a burning sensation in the nose and throat, which would have resulted in a movement of the head if inhalation had been continued longer. Very singularly, this subject could inhale oil of mustard for a considerable length of time without any uiscomfort if the cone was held at 4 or $5 \mathrm{~cm}$. from the nostrils. In fact, the change in respiration appeared before the sensation was detected.

Blood pressure tests. The subjects were the same as were used in the previous respiratory tests. It was found that the blood pressure of one subject engaged in manual out-door labor dropped $10 \mathrm{~mm}$. after sitting quietly for an hour and from then on maintained a fairly constant level, so that all of the subjects were given an hour's rest before any readings were taken. The general technical procedure has been outlined in the introduction. As in the previous respiratory tests every attempt was made to exclude psychical stimuli from entering into these reactions. For example, it was found that the blood pressure from subject $\mathbf{S}$. not only reacted very strongly to the first inhalation of butyric acid, but also showed a similar blood pressure change for every substance inhaled thereafter. The subject felt that he could still detect the butyric acid which was extremely repugnant to him. In view of this observation the agreeable odors were tested first, then the mild irritants, the disagreeable odors and finally the strongly irritating substances. As stated in the introduction only a change of $2 \mathrm{~mm}$. or more in blood pressure during the inhalation of a vapor was considered of any significance.

A summary of various readings in the different subjects is shown in table 1.

Carotid pulse tests. A large number of graphs were obtained from the same subjects as were used for the respiratory and blood pressure tests, including the anosmic and my son asleep and awake. The same vapors were used for the inhalations.

When the pulse waves from these tracings were counted and their height measured before, during and after stimulation, the conclusion was reached that these data are of little comparative value and are therefore not included in this report. It is true that many graphs taken during the inhalation of benzol, ether, chloroform, formalin, ammonia, etc., show changes in the height and rate of the waves during the inhalation period. It is also true that the same vapors frequently do not alter the pulse in the same individual. Pulse tracings taken during the inhalation of the very irritating substances usually show abrupt changes in the height and depth of their waves which are the result of swallowing or other movements of the head and neck. 


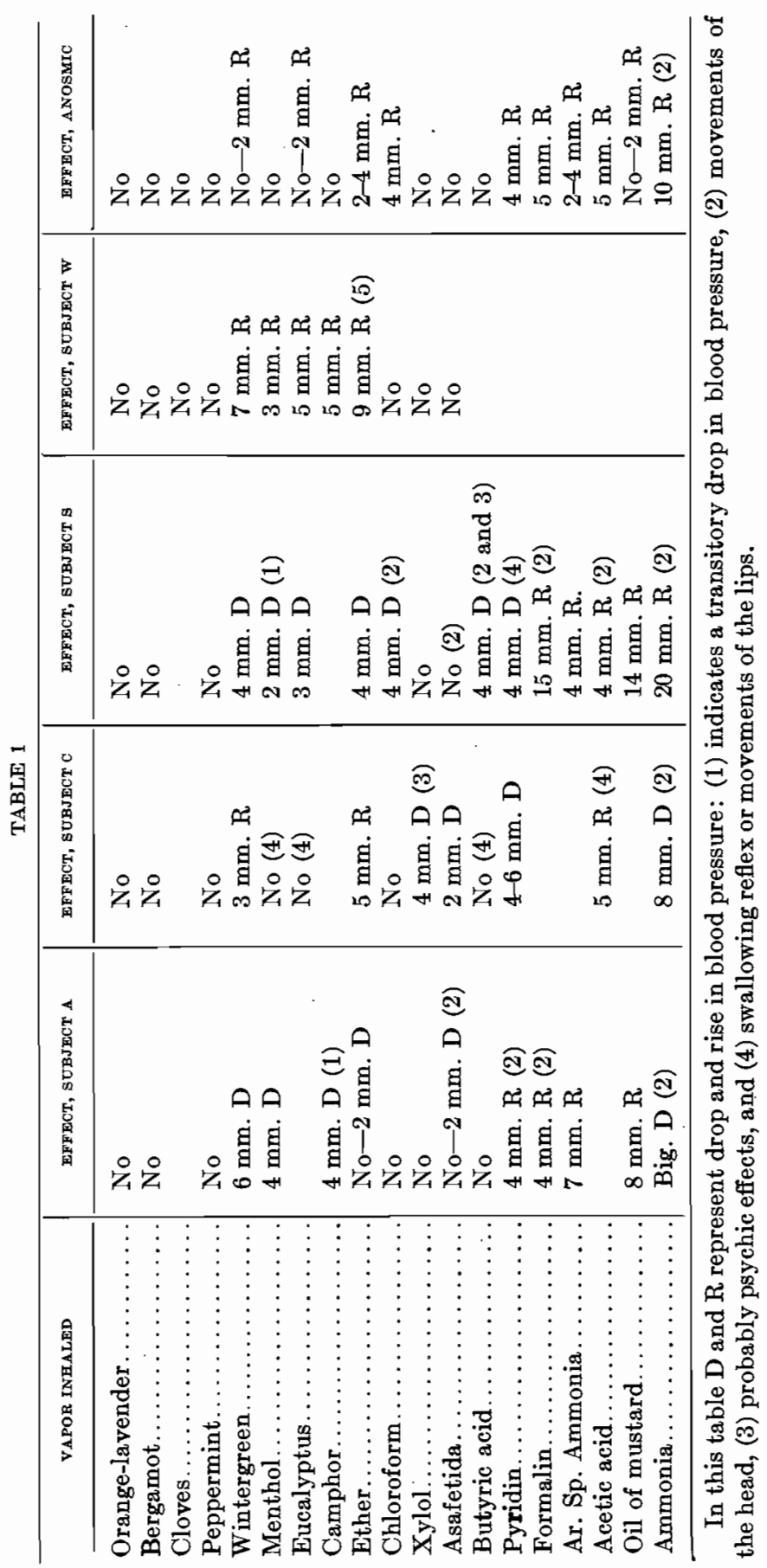


Several points of general interest were noted when the tracings were taken on my son while he was asleep. As was the case during the respiratory tests, inhalation of the agreeable, disagreeable and mildly irritating substances did not awaken the lad, but on the other hand, the first inhalation of ammonia awoke him immediately. It was observed in these tracings that his pulse became arrhythmical for some time after inhalation of several agreeable and disagreeable odors and xylol and benzol. This may be comparable to the so-called pseudo-vagal reaction frequently obtained in rabbits after fatigue from much inhalation or from several inhalations of benzol.

Some plethysmograms were taken from the forearm of the anosmic during the inhalation of several vapors, but independent movements of his arm produced so many irregularities that these records could not be used.

Respiratory tests on subjects under light ether anesthesia. Through the courtesy of Doctor Cliff, Director of Multnomah Hospital, the writer was permitted to make these inhalation tests on a number of surgical cases immediately after operation. The subjects selected were free from upper respiratory infections and were not likely to suffer shock from the operations. Each subject is referred to by number - 1, a young woman (Bartholin cyst), 2, a young man (osteomyelitis of the femur), 3, a young man (osteomyelitis of the humerus), 4, a negro boy (skin graft on arm), 5, middle-aged man (bone graft) who was deeply under ether after the operation and showed signs of shock, 6 , young woman (salpingectomy), 7, young woman (appendectomy).

Directly after the operation the patient was removed to a warm room, where thoracic respiratory tracings and systolic blood pressure readings were taken during the inhalation of oil of cloves, asafetida, wintergreen, xylol and ammonia. It was found that if a respiratory reaction is to be obtained from odors the subject nust not be too deeply under the influence of ether or under what may be a combination of ether and natural sleep.

The respiratory graphs from all subjects obtained during the interval of inhalation of very strong concentrations of ammonia (fig. $3 \mathrm{~A}$ from 2) disclosed a complete suspension of respiration. Weaker concentrations produce a marked depression and slowing of the respiratory movements. Of the five subjects tested, wintergreen ordinarily produced the next strongest alteration of respiration, always causing a marked inhibition. The excursions in a graph from subject 2 (fig. 3 B) exhibit a shortening of the inspiratory and expiratory phases, the rate remaining unchanged. In subject 1 it evoked a slowing of the rate as well as a shortening of the excursions and in subject 4 respiration was brought to a standstill.

Xylol varied in its ability to depress respiration but its effect is almost nil when compared to its effect in rabbits. The xylol tracings from subjects 2,5 and 6 are very similar to the asafetida tracing of figure $3 \mathrm{C}$. The 
excursions portray only a slight depression which affects both the inspiratory and expiratory phases. The depressive influence is more marked in the tracings from subjects 1 and 4, while there was no change in respiration in subjects 3 and 7 . The only suggestion of any slowing of respiration occurs in the tracings from subject 1.

Respiratory tracings obtained during the inhalation of the disagreeable odor asafetida reveal only a very slight depression of the excursions in subjects 2 and 7 (fig. $3 \mathrm{C}$ from 2). In subjects 3 and 4 the depression is slightly more pronounced, taking place in the expiratory phase of the excur-

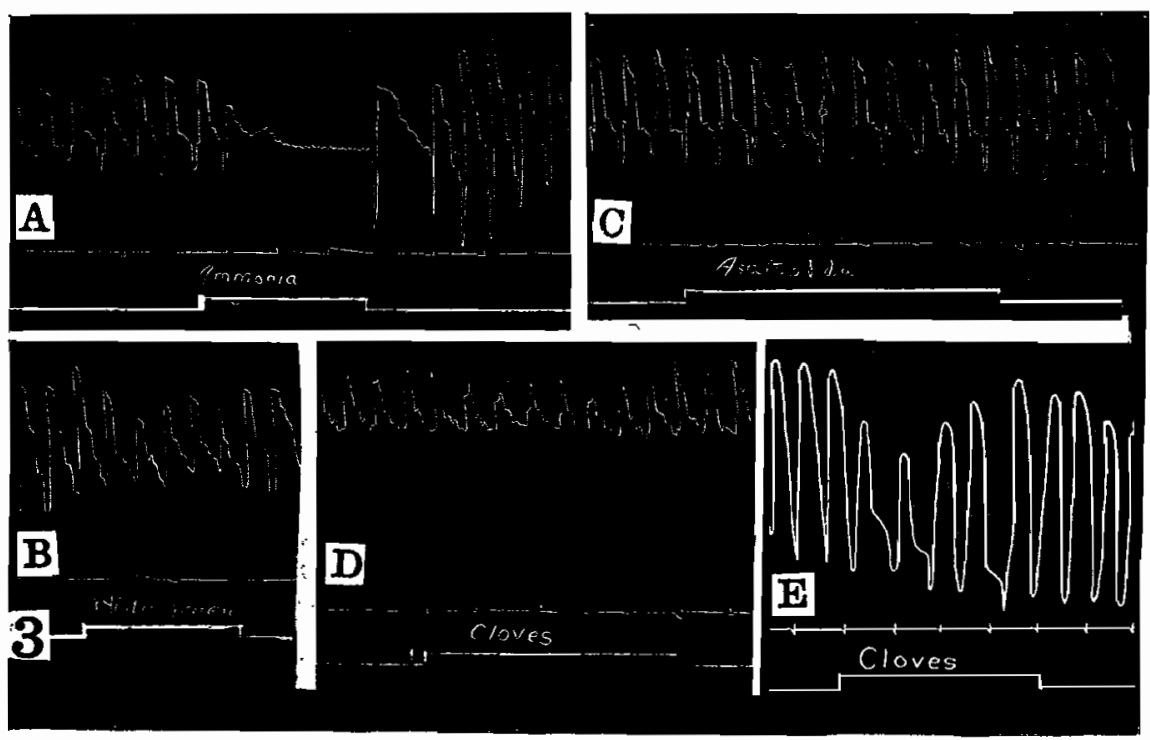

Fig. 3. Thoracic respiratory tracings from anesthetized subjects 2 and 1 , inspiration upstroke, time in 5 seconds; $\mathrm{A}$, ammonia tracing from $2 ; \mathrm{B}$, wintergreen from 2; $C$, asafetida from $2 ; \mathrm{D}$, oil of cloves from $2 ; \mathrm{E}$, photographic print of a tracing made from a non-contrasty oil of cloves graph from subject 1; the gradual drop throughout the tracing was caused by a small leak in the balloon.

sions in 3 and in the inspiratory phase in 4 . In 4 the expirations are somewhat deepened. Respiration is unaltered by asafetida in subjects 5 and 6 , the former was deeply under the anesthetic and the latter may have passed into a natural sleep when this tracing was taken. Unfortunately no test was made of asafetida in subject 1 , whose respiration was always slowed as well as shortened by the other inhalants.

Oil of cloves, which was inactive in the anosmic and is a specific olfactory inhibitory stimulant in some rabbits, produced an inhibitory effect on respiration in all subjects but 5 . The lack of response in this subject may be attributed to the depth of the anesthesia and shock. Figure $3 \mathrm{D}$, an oil 
of cloves tracing from subject 2 , shows a shortened inspiratory phase of the excursions with no change in their rate. The oil of cloves tracings from subjects 3, 4, 6 and 7 disclose about the same amount of depression of their excursions as is portrayed in figure $3 \mathrm{D}$. In subject 3 the depression is one which involves the expiratory phase of the excursions. Figure $3 \mathrm{E}$, which is a photographic print from a tracing of a non-contrasty oil of cloves graph from subject 1 , shows a slowing of the respiratory rate in addition to the usual shortening of the excursions, apparently causing a reduction in ventilation proportionate to that obtained in rabbits with this substance.

Several systolic blood pressure readings were taken from the earlier anesthetized subjects before and during the period of inhalation. So many independent changes were observed in the pressure before the inhalations were given that these tests were discontinued.

Comments. Previously reported experiments on rabbits have shown that the respiratory and vascular changes obtained during the inhalation of the odoriferous or irritating vapors are produced chiefly through stimulation of the olfactory and trigeminal nerve endings in the nasal mucosa.

Ponzo, Russi, and Malan have independently studied the effect of inhalations of several substances in man. I have not seen Ponzo's and Russi's papers, but Malan states that Ponzo advised his subjects as to the nature of the experiment and explained to them that they were to breathe deeply at a given signal and continue to do so until told to stop. His quoted results show that agreeable substances permit of deep inspirations, while disagreeable ones cause irregularities. Malan quotes Russi as concluding that agreeable substances produce prolongation of the inspiratory phase and disagreeable ones act on (lengthen?) the expiratory phase.

Malan used a very different procedure than Ponzo. His normal subjects, chiefly soldiers, were blindfolded and were not told what was to be done to them. Pneumograms and sphygmograms were taken during the inhalation of the essence of violets, scatol and acetic acid. This author found that each individual reacts in a different way to these substances, there being no constant relation to the stimulus employed. Violets produced acceleration of respiration in 15 per cent of the cases, inhibition in 5 per cent and no change in 80 per cent; scatol caused acceleration in 25 per cent of the cases, inhibition in 13 per cent and no change in 62 per cent; while acetic acid resulted in acceleration in 25 per cent of the cases, inhibition in 7 per cent and no change in 68 per cent. Concerning the pulse changes violets produced acceleration in 64 per cent of the cases, a slowing in 13 per cent and no change in 23 per cent; scatol evoked an acceleration in 53 per cent of the cases, a slowing in 26 per cent and no change in 21 per cent; acetic acid caused an acceleration in 49 per cent, a slowing in 9 per cent and no change in 42 per cent.

It is apparent from the foregoing inhalation-respiratory tests on unan- 
esthetized normal subjects that the writer's results do not accord with the "laws" of Ponzo or of Russi, neither do they show the same irregularities or as many negative reactions as Malan portrays. It would seem to the writer that Malan's procedure would be especially adapted to produse psychic impulses from fear and surprise, which would invalidate his results as reflex effects. The writer always obtained a very definite inhibitory response from inhalation of the powerful irritants such as acetic acid and ammonia, strong concentrations usually producing a complete arrest of respiration. Two types of altered respiration result from inhalation of the mild irritants and the agreeable and disagreeable odors in normal unanesthetized subjects-1, a shallow type, consisting of shortened inspirations or a mixture of shortened inspirations and deeper expirations, usually accompanied by some increase (sometimes no change or a decrease) in the rate of the excursions; 2 , a deep type, usually showing a decrease (sometimes no change) in the rate of the excursions. The first type should ordinarily contribute to a decreased ventilation, while the second type would probably cause little or no change. Ordinarily the same subject will react about the same for a given substance, but tracings from the same individual have shown type 2 reaction one time and type 1 or a combination of 1 or 2 another time.

In unanesthetized subjects blood pressure changes are less easily produced than in rabbits, and furthermore, they are not always a rise in pressure. No changes occurred in my subjects from inhalation of the agreeable odors and in some individuals from the disagreeable odors and some of the mild irritants. In other subjects there was a change in blood pressure during the inhalation of the disagreeable and some or all of the mild irritants, which may be a rise in some instances and a drop in others. Wintergreen produced marked blood pressure changes in some subjects. All of the extremely irritating substances caused a change in blood pressure which was usually a rise. Aromatic spirits of ammonia always caused a rise in my subjects, while ammonia itself sometimes brought about a drop.

The respiratory tracings obtained from my anosmic are only in partial agreement with Malan's 30-year-old anosmic. The latter is reported as giving no respiratory changes from inhalations of violets, scatol and acetic acid. In my subject the respiratory and blood pressure responses to the strong irritants, excepting oil of mustard, and to most of the mild irritants were only slightly weaker than was the case in the normal subjects while all of the odors and xylol did not alter blood pressure or respiration.

Since the respiratory changes were practically the same in all of the anesthetized subjects and this reaction is identical to the reaction in rabbits, namely, inhibition caused by a shortening or a shortening and a slowing of the excursions, this inhibitory action is thought to be the respiratory reflex in man resulting from stimulation of the olfactory and trigeminal 
endings in the nasal mucosa by the inhalants; while other alterations of respiration frequently obtained in unanesthetized subjects may result from psychic stimulation or other reflexes. It appears impossible to entirely exclude the aforesaid factors in unanesthetized subjects.

It would be of interest to know how and where sleep blocks the olfactory and the trigeminal respiratory reflexes.

\section{SUMMARY AND CONCLUSIONS}

In normal unanesthetized subjects the inhalation of the strong irritants produces marked depression and slowing or complete arrest of thoracic respiration. The thorax generally becomes much more contracted.

Mild irritants, disagreeable and agreeable odors cause either a considerably lowered inspiratory phase and an increase (sometimes no change or a decrease) in the rate of the excursions or a considerably deepened inspiratory phase and a decrease (sometimes no change) in the rate of the excursions, A few tracings show a combination of these two reactions. Frequently there is some effort toward deepened expirations in case of the disagreeable odors and mild irritants, where the response is much stronger than it is with the agreeable odors.

In normal unanesthetized subjects the systolic blood pressure is unaltered by inhalation of the mild agreeable odors. Disagreeable odors and mild irritants may or may not alter blood pressure and the change may be a rise or a drop. Strong irritants produce considerable change in blood pressure,
usually a rise.

Respiration of the anosmic shows no changes from the inhalation of the agreeable and disagreeable odors or of wintergreen and xylol. Peppermint, menthol, eucalyptus, chloroform, ether, benzol, weak concentrations of the strong irritants and strong concentrations of the vapor from oil of mustard elicit a deepened inspiratory phase and a reduced rate of excursion. Strong concentrations of the very irritating substances, excepting oil of mustard, induce a considerably shortened inspiratory phase and some
increased rate of excursion.

The changes in blood pressure of the anosmic during inhalations follow very closely those of respiration. Some mild irritants although not detected by the subject effect a change in respiration and blood pressure.

A long series of inhalations of various agreeable and disagreeable odors and mild irritants failed to awaken a sleeping subject or to produce any respiratory changes. The first inhalation of strong ammonia for over 20 seconds in one test evoked no respiratory response and did not awaken the
subject.

Respiration in several subjects under light ether anesthesia responded to five inhalants as follows: Oil of cloves usually produced some depression or depression and slowing of the excursions, with one exception this depres- 
sion affected only the inspirations. In those subjects where asafetida and xylol react, the effect is very similar though often weaker than oil of cloves, and the inhibitory influence of xylol is decidedly weaker than in rabbits. Wintergreen usually evoked a similar but much stronger inhibitory action than oil of cloves while strong concentrations of ammonia generally brought respiration to a standstill.

Blood pressure in anesthetized subjects presented so many inconstant and rapid changes that readings taken during the interval of an inhalation were of no value.

The inhibitory respiratory reaction obtained from inhalants in human subjects under anesthesia and in some records from non-anesthetized subjects is apparently the olfactory-trigeminal respiratory reflex from inhalants in man. This is identical to the olfactory-trigeminal reflex in rabbits. Different respiratory changes sometimes occurring in unanesthetized subjects may come from psychical stimulation.

\section{BIBLIOGRAPHY}

Crostand, Goodman and Hocket. 1926. Journ, Exper. Psychol., ix, 398.

Matan. 1925. Arch. internat. de laryngol., iv, 666.

Ponzo. Cited by Malan.

RUssi. Cited by Malan. 